\title{
Problems of Formation of Diagnostic Features in the Diagnosis of Aircraft Engines
}

\author{
V. I. Pronyakin ${ }^{1, *}$ \\ ${ }^{1}$ Bauman Moscow State Technical University, 105005 Moscow, Russia
}

\begin{abstract}
The article is devoted to the evaluation of current technical condition of aircraft engines. Deals with the choice of the detection method of diagnostic features required for degradation assessment, emergency protection and detection of incipient defects on the example of cyclic machines and mechanisms. For the formation of diagnostic features in the diagnosis of aircraft engines use different physical effects (vibration, shock, heat radiation, electrodynamic and thermal processes, wear debris in oil, etc.). Classification of defects and requirements for the development of diagnostics systems is formed based on them. The article describes the requirements for diagnostic signs. The article provides a promising phase method that allows obtaining stable diagnostic characters in exploitation. The result of applying the method is shown. Diagnostic signs are formed. In mathematical modeling it was used the traditional theory of the description of rotary mechanisms. The data obtained are compared with experimental data.
\end{abstract}

\section{Introduction}

Currently, the task of assessing the technical condition of mechanical and electromechanical systems and predict their trouble-free operation is solved, as a rule, on the basis of a large volume of statistical data and failure analysis.

For complex technical objects (gas turbine engines, turbines, etc.) are dominated by expert assessment, which are determined by technical level experts.

This article focuses on the consistent diagnostic features necessary for the registration of the degradation and identification of incipient defects in functional diagnostics.

To assess the technical condition used the regular parameters and physical effects that occur in the process of working of gas turbine engine (GTE) (vibration, shock, heat radiation, electrodynamic and thermal processes, the products of wear in the oil, etc.).

It should be noted that these physical phenomena for high-speed engines are slow.

The processes associated with rotary engine, proceed quickly. The evaluation, built on the operating elements of the device failure, do not provide reliable emergency protection. The refusal of the engine during flight is unacceptable. For the exception of emergency needs to be performed on the ground assessment of the current technical condition and to provide reliable emergency protection in flight. While the kinematic parameters of movement of the machine elements resulting from the worker processes, usually for diagnostic purposes are not controlled. Possibilities of widely used parametric diagnostics grow due to the increase in the number of controlled parameters and increase the potential of computing machinery. Regular parametric diagnostics registers are already visible deviations from the standard values in the machine. This demonstrates the need of prevention or repair.

Currently, the diagnoses of GTE are widely used statistical methods, analysis of failures and their consequences for risk assessment. Life classification of defects and development of requirements for a diagnostic system are running on this database. Also used maintenance based on reliability theory. However, the statistical definition of probability of failure does not guarantee complete safety (for example, GTE in aviation). It is necessary to carry out continuous monitoring of current technical condition and reliable emergency protection complex and expensive technical objects.

The analysis of the traditional methods of obtaining information on the operation of the rotary mechanisms, including the vibration analysis showed that the main reasons for the lack of effective informationmetrological maintenance of operation of machines and mechanisms include the following factors:

1. Methods of amplitude (e.g., vibration monitoring) to obtain information to solve the problem of assessing the current technical condition, the problem of the guarantee of emergency protection. Methods do not allow changing from the system of preventive maintenance systems the maintenance to systems on the current technical condition.

2. The metrological level of the measuring instruments used for diagnostics of machines and mechanisms, currently, medium, or low. In practice of metrological assurance, relative accuracy is a few percent. In this regard, the necessary informative content

* Corresponding author: vip-u@,yandex.ru 
of measurement information is not provided. Monitoring characteristics of vibro-acoustic processes is the main approach in the diagnosis of operating machines and mechanisms. Assessment of the vibration state of large machinery, identify the sources of vibration and its maximum remains fundamentally important, but unresolved to the full task. Low metrological level of the results of measurement of vibration parameters (the relative error from 1 to $10 \%$ ) allows to obtain informative quantitative individual characteristics required to assess the current technical condition, determining incipient defects and prognosis. Therefore, it remains a problem obtaining a stable diagnostic characters (or slowly changing) to assess the performance of rotor systems at all stages of the life cycle. Vibration measurement for functioning mechanisms with distributed mass allows obtaining statistical characteristics. Automatic detection of individual defects implemented. Applied monitoring systems measure the current values of parameters and provide automatic responses when thresholds are reached.

Expert evaluations prevail at the present time. They are subjective in nature. Assessments do not provide the effective operational solutions to perform the control actions of emergency protection.

3. Effective diagnosis of low speed and low-speed mechanisms with low vibration, high-speed systems and devices with distributed mass, etc. is not provided. The information collection system, registered vibroacoustic processes are improved. Methods of information processing are improving mostly.

4. Determining the relationship between measurements and peculiarities of functioning of the structural elements is a fundamental problem of traditional methods. Reliable correlation between measurement results and design, to obtain a quantitative estimation of the degradation components of the device, using the applied physical quantity (e.g., velocity and vibration acceleration), is unable to install. In this regard, the creation of automated and automatic systems of diagnostics and emergency protection of the functioning of machines and mechanisms remains an important issue.

Stage classification of the defects, currently implemented in the analysis of failures and technogenic accidents in the process of developing methods and tools for assessment of technical state of machines and mechanisms, is required. The detection of defects and determination of diagnostic features is made on the basis of the obtained results. Mathematical modeling of mechanisms using computational experiments for the simulation of defects are used to a lesser extent in the practice of diagnosis.

5. In the analysis of rotary mechanisms are widely used spectra of oscillatory processes. Spectra of oscillatory processes provide basic diagnostic information, but have a weak immunity. The main problem the most common amplitude methods is that the acoustic signal contains all the information about the interaction of parts of the working mechanism, including its natural vibration background. But how to retrieve information about a particular node, how to localize failures and to obtain quantitative characteristics of the changes - that's the question. The complexity of the defects is determined by high level of noise and a small change of the useful signal. The structure of the spectra changes over time (particularly for long intervals of use) in connection with a radical change of vibration parameters and their use for assessment of the evolution of the device, its technical condition, obtain trends, and forecast is very problematic. As a rule, a significant increase in the overall level of the signal or its individual components says only gross changes in the condition diagnosed mechanism leading to the loss of its functionality. The localization of developing problems requires the determination of the relationship of vibroacoustic signal with the kinematics and dynamics of structural elements of the cyclic mechanism. There are positive results for concentrated systems (for example, a bearing). The main problem is distributed mechanical and Electromechanical systems (e.g., turbo - and hydroelectric generators, gas turbines, etc.).

Thus, in connection with the with-ranks of failed traditional methods and means to fully solve the problem of obtaining information about features of rotary mechanisms and to solve the problem of obtaining stable diagnostic features.

\section{The requirements for diagnostic signs}

Obtaining information about the functioning of the device is the main task of technical diagnostics. Processing of results of measurement of various physical quantities, selected in connection with the task, is a source of information. Thus it is necessary to solve a number of problems related to the task and metrological support and to determine:

- measurement accuracy associated with the level of required technical information,

- primary transducers with operating conditions, and testing;

- number of measuring channels

- the relationship of the measurement data with the design and controlled process product

- algorithms of processing of measuring information etc.

The selected method of diagnosis determines the controlled physical quantity, measuring instruments and methods of mathematical processing of measurement results to correlate them with the design of controlled nodes and identify diagnostic features. Currently, the analysis of failures and identification of their Association with defects of the device are performed. It's a long process, which is unacceptable to ensure troublefree operation of complex and expensive technical objects. Analysis of the impact of failures, breakdowns, industrial accidents and catastrophes runs of the decade, but to solve the problem of estimating the current technical status effectively fails. 


\subsection{The traditional approach}

It is accepted that in systems of technical diagnostics use the principle of deviations (principle of Salisbury), and therefore it is proposed to use the characteristics of a "reference product or a mathematical model of the standard." However, the inevitable spread of parameters due to manufacturing errors, Assembly, adjustment and the impact of external influences. When high measurement accuracy is necessary to obtain detailed information, register the variation of parameters and therefore, the "reference values" may not have nominal value. In this connection there are ranges and algorithms that contain a number of consistent characteristics for the evaluation of the mechanism work. Currently various diagnostic methods are being intensively developed (neural networks, statistical analysis, information theory, theory of pattern recognition, the Bayesian method, etc.). But they do not provide a solution to the problem of assessing the current technical condition of operating machines and mechanisms. Statistical diagnostic methods and the Bayesian method, implemented on the basis of statistical information. They have different rules in finding the best solution and bound to the statistical structure of the diagnostic data [1]. However, the use of these methods for detection of defects does not provide quantitative individual characteristics of a serial product sample to assess the degradation, the development of defects and prognosis.

For information on the functioning of the GTE, it is primarily necessary to identify any stable diagnostic features with invariance and noise immunity to external influences. It is necessary to determine the diagnostic characteristics that accompany the operation of the facility over the life cycle, and provide information on specific device nodes. The diagnostic performance of symptom connected in a controlled setting, sensitivity, accuracy and invariance, as well as the possibility of implementing quantitative analysis, the use of classical methods of mathematical modeling of mechanical and electromechanical systems and forecasting. Basically, the amplitude method is used in the diagnosis of GTE to obtain the measurement information. In accordance with that defined by the controlled physical quantity, requirements of accuracy of measurements and mathematical processing. In the vibration (the amplitude method) is velocity, vibration acceleration and amplitude. These parameters carry information about all the interacting elements of the device, but sensitive to external influences and changes modes of operation. The problem is obtaining quantitative information about the degradation of specific parts of the mechanism. Also there is a background vibration characteristic of defectfree product and, therefore, much more complicated detection of incipient defects. Also used phase method, in which the monitored characteristics associated with the operational cycle of the device.

\subsection{The requirements for diagnostic signs}

The requirements for diagnostic symptom do not depend on a method of obtaining information, selecting the measured variables and formed by the next OS main conditions:

1. The measured physical quantity to the maximum extent depends on the interaction of structural elements and processes in the device, and to a much lesser extent from external influences and modes of operation.

2. Optimally, when the scan characteristic has the invariance and stability to various influences in the process of operation and to change modes of operation. The main quality characteristics during the entire life cycle, enabling the comparison and quantification of changes in the mechanism, should be preserved $[2,3]$.

3 . The selection of the measured physical quantity and the accuracy of the transducers determine the possibility to quantify the slow processes that characterize the degradation mechanism, deterioration and identification of incipient defects. An error in the relative-ness of vibro-acoustic parameters from $0.1 \%$ to $10 \%$ corresponds to the level of gross errors. The measured physical quantity should be in the maximum degree does not depend on external influences, changes the modes, which is necessary for maximum repeatability of the diagnostic signs.

An example is the measurement of time intervals corresponding to the turnover and its shares, for a shaft turbine TPS at check 3 shutdowns of the generator of the turbine unit from an external network, showed repeatability of chronograms, providing analysis of the mode (Fig. 1) [6].

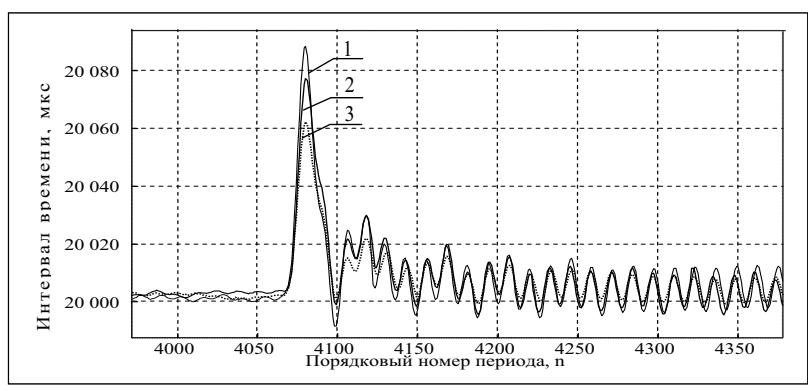

Fig. 1. The chronogram generator shut-down of turbine CHP plant in different modes from the external network

4. Should be provided with the quantitative correlation characteristics of the diagnostic signs of degradation of product design and processes in a functioning device. Due to problems binding the measurements to the design of mechanisms in technical diagnostics is dominated by peer review. With the use of vibroacoustic parameters, the main problem is the relationship of the controlled parameters (velocity and acceleration) solving a specific problem, for example, the definition of wear of the gear teeth in the gearbox, the quantitative wear of the bearing. An unsolved problem for large-sized products is to identify the sources of vibration.

5. Identify diagnostic signs should be extended to standard product without the need for adaptation to a particular series of condom sample.

Currently developed approaches to the definition of requirements for diagnostic systems, but remains 
unresolved the question of determining the estimation algorithm of operation of the device, detection of defects and prediction of their development. It is proposed that the use of statistical information on the work of the diagnostic mechanism will allow to assess to confidence level of the diagnosis of mechanism.

\section{Metrological level}

The metrological level of the measurement results should provide an opportunity to quantify changes in the mechanism in operation. The measured physical quantity and the accuracy of the transducers should be sufficient to record small changes of the controlled parameter characterizing the wear, degradation mechanism and incipient defects. To empower solutions to the above outlined problems for rotor systems proposes a phasechronometric method of diagnostics of cyclic mechanisms [4-6], in which to perform the analysis of kinematic parameters of motion of the device in combination with mathematical modeling on the basis of the classical theory of machines and mechanisms to identify diagnostic signs of defects. The kinematic parameters of the working cycle of the machine, the nature of the interaction which persists throughout the life cycle, are controlled by using phase methods. Since the operating cycle has remained relatively stable in the functioning of the product, there are conditions to obtain stable diagnostic characteristics observed during the life cycle. For detail information and obtain the numberbased estimates of changes in the mechanism provided the necessary metrological level (- relative error of $5 \bullet 10$ $4 \%$ at the industrial frequency of $50 \mathrm{~Hz}$ ).

\section{Mathematical modeling}

The main feature of phase methods is the ability to use the classical theory of the description of machines and mechanisms, developed for a variety of cyclic mechanisms specialists in a specific area of technology to analyze the device and determine diagnostic features. The measurement information has a significantly smaller noise in comparison with the natural background vibrations of machines and mechanisms, what allows going to the level of incipient defects. The capabilities of computers allow the development of more detailed mathematical models for diagnostics and carrying out of computing experiment on revealing of diagnostic signs. The result of mathematical modeling is measuring information corresponding to experimental data, and there is a possibility of simulation of defects and classification of defects at the stage of creation, testing and production.

The mathematical model corresponding to the accurate measurements obtained by using high-precision measuring systems is a key factor of communication measurements with the processes of functioning of parts and components in the device. The detail of the results of computational experiment should correspond to the results of processing of experimental data obtained in precision measurements. For example, the comparison of mathematical modeling results and experimental data of functioning of the cutting machine, taken with a rotating spindle in the system drive-reducer-spidel presented.

\section{The results of the comparison of mathematical simulation and experiment}

An example of this approach is the creation of measurement monitoring of turbine TPS with the implementation of the diagnostic system and mathematical models of turbine T-250. The measuring system provided absolute error of measurement of time intervals corresponding to the turnover and its share in operating conditions $\pm 1 \cdot 10^{-7} \mathrm{~s}$, which corresponds to turbine relative error of $5 \cdot 10^{-4} \%$ of the nominal period on industrial-Thoth $[2,6,7]$. Spectral analysis is widely used to identify diagnostic features. In [8] describes the methods of recovery and interpolating the signals of the Fourier spectra. The spectrum parameters of torsional vibrations obtained by consecutively processing of the registered time intervals, sustainable, qualitatively and quantitatively change during the operation process, therefore suitable for the detection of diagnostic features.

Processing of the results of successive measurements of the respective revolution of the shaft and its share, built over a long period spectra of torsional vibrations of a shaft of the turbine (TA) T-250 [2] has preserved its major characteristics until now (Fig. 2).

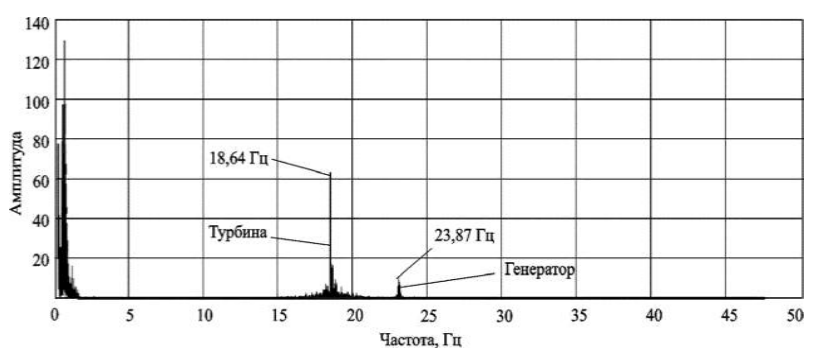

Fig.2. The range of torsional vibrations obtained from the experimental data for the turbine T-250

A mathematical model is developed to relate measurements of time intervals corresponding to a revolution of the shaft and the lobes, with structural TU elements. According to the results of the computational experiment produced a range of fundamental natural frequencies KRU-telnyh vibrations of a shaft (length 46 m, weight 130 tons, $3000 \mathrm{Rev} / \mathrm{min}$ ) (Fig. 3).

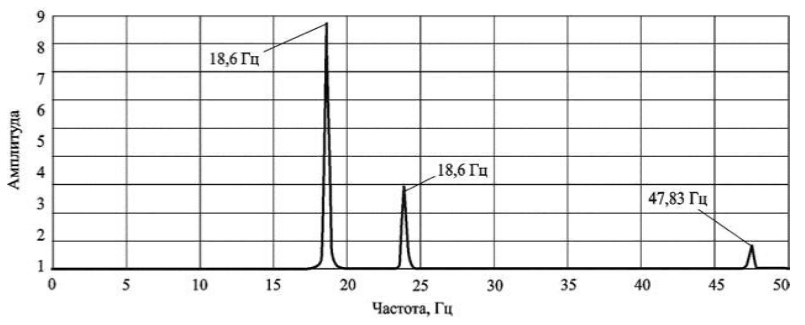

Fig. 3. The range of torsional vibrations obtained by mathematical simulation for the turbo unit T-250. 
Comparison of results of mathematical modeling for fixed frequency $18,60 \mathrm{~Hz}$ and of $23.87 \mathrm{~Hz}$ with the results of experimental data processing of $18.64 \mathrm{~Hz}$ $23,20 \mathrm{~Hz}$ showed differences ranging from 0.2 to $2.9 \%$, which allows to model defects and shape classification of defects using mathematical models in a phasechronometric performance.

The developed mathematical model [9] for turbine T250 takes into account the relationship between the electrical parameters of the generator, geometrical and physical-mechanical parameters of the turbine, including the moments of inertia and rigidity of the connected sections of the shafting.

On the one hand, the mathematical model provides an interpretation of the experimental data and classification of defects at the stages of development, testing and production. On the other hand, the mathematical model allows taking into account the individual characteristics of the system turbine-generator (moments of inertia, stiffness, dimensional and mass characteristics, the electrical parameters of the generator). This provides the possibility of measuring and computing support electromechanical systems throughout the life cycle and determining the deviation of system parameters based on the identified diagnostic features. Part of a mathematical model THAT includes the description of the turbine and generator required for describing TU torsional vibrations, bending vibrations of a shaft of the turbine, parameters of Electromechanical processes occurring in the generator in the longitudinal and transverse axes rigidly associated with the rotor.

To perform work on the turbine $\mathrm{T}-200$ in the mathematical model of TU T-250 was introduced, the original data is TU T-200 and pre-defined frequency of torsional vibrations of the shafting. After performing the experimental work was the comparison of the obtained experimental results with preliminary theoretical calculations.

According to the results of numerical experiments a spectra of the main natural frequencies of torsional vibrations of a shaft of the system generator-turbine was constructed. The experimental spectrum of frequencies of torsional vibrations of a shaft of TU T-200 obtained after further processing.

Comparison of results of mathematical modeling with the results of experimental data processing shows matching the frequency spectra obtained from periodogram of rotation of THE shaft and using a mathematical model.

The range has been pre-calculated using the mathematical model for TU T-250 and inputs TU T-200.

Its parameters differ from the experimental data for fundamental frequencies and 38,78 i.e. $45.98 \mathrm{~Hz}$ respectively from $0.39 \%$ to $1.20 \%$, which testifies to the effectiveness of the phase approach for the analysis of cyclic mechanisms (including GTE) and identify diagnostic features.

\section{Conclusion}

Thus, the application of phase methods for assessing the current technical condition and emergency protection of aircraft engines will provide conditions to obtain a stable diagnostic criterion. In combination with mathematical modeling on the basis of the classical theory of machines and mechanisms and the high metrological level of realized correlation of measurement results in the design to solve specific problems and achieve incipient defects.

\section{References}

1. O.F. Mashoshin, Diagnosis of aviation technology (information framework) (Moscow, MGTUGA publ., 2007)

2. M.I. Kiselev, A.P. Kozlov, A.N. Morozov et al., Izmeritel'naia tekhnika, 12, 28-29 (1996)

3. M.I. Kiselev, V.I. Proniakin, Nauka i obrazovanie. MGTU im. N.E. Baumana, 5 (2011) http://technomag.bmstu.ru/doc/192225.html

4. V.I. Pronyakin, Measurement Techniques, 51(10), 1058-1064 (2008)

5. V.I. Proniakin, Mir izmerenii 9, 57-61 (2011)

6. A.I. Baikov, M.I. Kiselev el al., Gidrotekhnicheskoe stroitel'stvo, 2, 2-8 (2015)

7. M.I. Kiselev, V.I. Pronyakin, Ya.V. Chivilev, N.A. Zroichikov, Thermal Engineering, 53(11), 868-872 (2006)

8. L.A. Aizenberg, B.A. Kravtsov, Avtometriia. 1, 6064 (1989)

9. A.S. Komshin, Measurement Techniques, 56(8), 850-855 (2013) 